\title{
Suggestions for Application of Artificial Intelligence in Pulse Feeling
}

\author{
Tong Zheng Hong* \\ As You Wish Healthcare Institute, Taiwan
}

Submission: January 24,2019; Published: March 08, 2019

*Corresponding author: Tong Zheng Hong, As-You-Wish Healthcare Institute, MS in Acupuncture awarded by National University of Health Sciences in IL, Taiwan

\begin{abstract}
Pulse feeling is one of the four skills in the traditional Chinese medicine (TCM) as the information for diagnosis and treatment, which seems to be the most possible way for the application of artificial intelligence in integrating traditional Chinese medicine with the Western medicine. However, it surely deserves much attention because the issues like the definition and the norm of the normal pulse, science-based evidence for the use of automatic sphygmography for the optimal decision-making method in face of subjective and objective information remain uncertain and need further research.

Keywords: Zeng (Pattern) identification; Pulse feeling; Artificial intelligence; Automatic sphygmography
\end{abstract}

\section{Introduction}

Zeng (Pattern), which is based on the information gathered from the fours skills of Looking, Smelling, Asking, and Pulse feeling, is the unique concept that distinguishes traditional Chinese medicine (TCM) and acupuncture from the Western medicine. Fundamental principles of exterior signs and symptoms in the TCM can reflect to some extent how internal organs function within the body, which is broader than the theories in the Western medicine. It is difficult for TCM physicians or acupuncturists in clinic to screen the information gathered through the four skills because there will be three confusing conditions:

I. Pattern corresponding to Pulse

II. Pattern against Pulse

III. selecting either Pattern or Pulse

In other words, TCM physicians or acupuncturists may face the challenges to pinpoint the key pattern for the best treatment outcomes. In the era of artificial intelligence (AI) progressing rapidly, it seems to be beneficial for the integration of AI with Pulse feeling in terms of saving time, costs and the best outcomes. However, this study aims to present that there are issues for TCM physicians, acupuncturists, and researchers to consider for the application of $\mathrm{AI}$ in the Pulse feeling skill.

\section{Look at AI and Pulse Feeling}

$\mathrm{AI}$ is recognized as "a system's ability to correctly interpret external data, to learn from such data, and to use those learnings to achieve specific goals and tasks through flexible adaptation" at present [1], which indicates that the data established for use is the key for applying AI to Pulse feeling. Pulse diagnosis is an extremely difficult and complex subject but important since it can provide very detailed information on the organs inside the body, reflecting the real conditions of the whole picture of concrete organs and abstract meridians. The pulse in the Western medicine is only viewed as a minor tool for diagnosis; however, Pulse diagnosis in the TCM plays one of the important roles for gathering information on

1) The balance between Zang-Fu organs in the body as a whole to get the whole picture of the vital substance like Qi, Blood, Essence, Yin-Yang, body fluids, and even the constitution.

2) The functions of the specific organ for the diagnosis based on the Five Elements.

Both TCM physicians and acupuncturists feel the pulses to discern width, amplitude, length, and particularly note the rate to differentiate the positions, the strength, and other qualities [2] 
which is different from observing the rhythm, intensity, rate, and whether any blood vessel tenderness, tortuosity, or nodularity in the Western medicine [3]. Information gathered from the Pulse diagnosis by the TCM physicians or acupuncturists can be complex and extremely subjective, even though there are in general 28 pulse qualities. Pulse in TCM bears the representations of Stomach qi, Spirit, and Root, which can be included in the data for diagnosis and treatment. However, it is truly difficult to define the normal pulse and interpreter properly Pulse without sufficient scientific evidence.

\section{Challenging Issues}

The information obtained from the four diagnosis skills can be divided into objective and subjective. Pulse-related information is viewed as subjective because it is abstract and judged only by TCM physicians and acupuncturists based on their experience, while objective information is gathered through Looking, Smelling, and Asking. Mai-jing (Pulse Classic) is one of the TCM classics on Pulse published in the 3rd century. In this classic, Floating (or called Superficial) pulse is defined as the pulse that is felt with light pressure but disappears with heavy pressure. The scenario of the Floating pulse is depicted as the trunk floating on the water. The TCM and acupuncture theories are analyzed and organized with the observations of the nature by the ancient masters, who are familiar with the inductive reasoning. Some of concepts are abstract because they are depicted with the observers' personal life experience. The first challenging issue is what the normal pulse really refers to. It is believed that the normal pulse can indicate the good state of Jing (Essence), Spirit, and Qi. However, the representations of Stomach qi, Spirit, and Root are abstract and obscure, though it is generally accepted that 4 beats per respiratory cycle means the presence of Stomach qi and accepted to be the normal pulse in the TCM [4]. On the other hand, Spirit is said to be the combination of Jing (Essence) and Qi. Spirit refers to the strength in the Pulse that is felt soft. However, how soft it refers to remains unclear. To what degree the health and normal pulse is can arouse controversy from the aspect of the quantitative research. In other words, sampling population is first concerned in the scientific research. Unfortunately, the definition of the "normal pulse" seems to be the mission impossible at present. The second challenging issue shows that all the descriptions of Pulse are indeed abstract to be interpreted accurately and challenge TCM physicians and acupuncturists. We can understand to some extent it is not easy at all to clarify how light or heavy this Pulse truly refers to, which can be only determined with the personal experience. The primary principle for TCM physicians and acupuncturists to follow in feeling the pulse is that they need to put fingers on the three pulse positions-Cun, Guan, and Chi at the radial artery of two hands simultaneously for the differentiation, feeling pulses presented in each position with light, median, or heavy pressures to make the differential diagnosis. This is the third issue that challenges those who follow scientific research methods to understand with automatic sphygmography $[5,6]$. As a matter of fact, what the specific pulse really refers to remains unreal and uncertain. The current automatic sphygmography cannot meet the clinical requirements because the real differences of pulse cannot be understood without light, median, or heavy pressures on each position on the two hands at the same time, which can surely make the information incomplete for the differential diagnosis. The fourth challenge issue TCM physicians and acupuncturists face is how to select the information obtained from the four skills for diagnosis and treatment. In a whole, there are several options for TCM physicians and acupuncturists to make a correct decision in clinic shown in Figure 1.

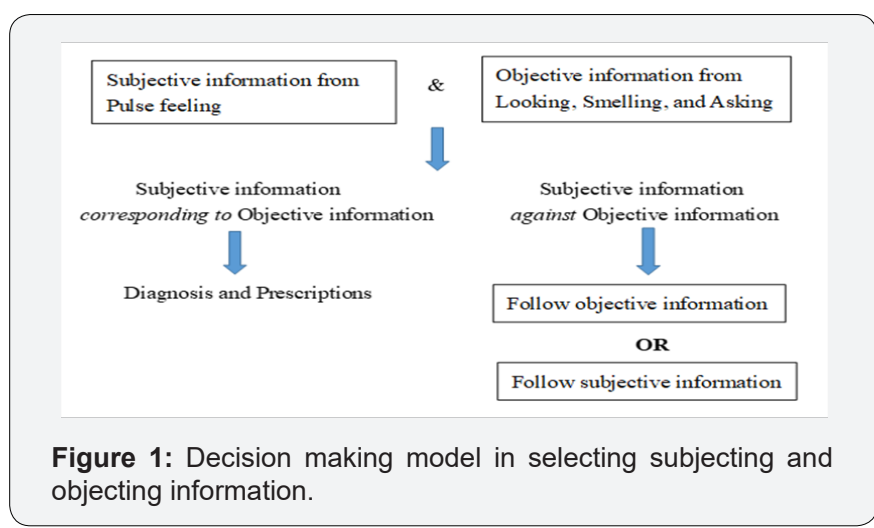

\section{Discussion}

Zeng (Pattern) identification plays a key role in the diagnosis and treatment, which relies on the accurate information gathered from the four skills. However, how to identify the correct Zeng indeed requires the experience of TCM physicians and acupuncturists. Pulse feeling is absolutely subjective and whether or not the pulses felt can really reflect the health condition can challenge TCM physicians and acupuncturists in making an accurate decision for diagnosis in clinic. It is for sure that the unexpected treatment outcomes may occur as long as TCM physicians and acupuncturists are not able to distinguish the minimal differences. Since the real meaning of the "normal pulse" in TCM is confusing and uncertain, it is absolutely controversial to the scientific researchers when they are making decisions. To some extent, this issue seems to be the top priority for the TCM physicians and acupuncturists to reconsider for the scientific research. With insufficient evidence gathered from the evidence-based researches, whether or not the current automatic sphygmography developed with the scientific and technical methods can be used to detect the real pulses corresponding to the patient's health state may remain uncertain. In other words, the information provided by the automatic sphygmorgraphy could be not authentic enough to be included into the AI database.

\section{Conclusion}

The dialogue between TCM physicians and acupuncturists and the scientific researchers is required for the integration of TCM and the Western medicine. However, we need to understand that the language barriers may hinder TCM physicians and acupuncturists and scientific researchers from getting the real 
picture of TCM. In addition to Pulse feeling, the information obtained from the other three skills needs scientific evidence as well to link TCM physicians and acupuncturists with scientific researchers, which deserves much more attention.

\section{References}

1. Andreas K, Haenlein M (2018) Siri, Siri in my Hand, who's the Fairest in the Land? On the Interpretations, Illustrations and Implications of Artificial Intelligence, Business Horizons 62(1).

2. Sacred Lotus Chinese Medicine. TCM Diagnosis by Palpation (Pulse Diagnosis) - One of the 4 Pillars.
3. Hal H. A Misguided Study to Test the Reliability of Traditional Medicine Pule.

4. Maciocia G (1994) The Practice of Chinese Medicine: The Treatment of Diseases with Acupuncture and Chinese Herbs.

5. Hsiao TC (2009) Multi-Channel Signal Processing Technology for the Modification of Automatic Sphygmography. Yearbook of Chinese Medicine and Phamacy. Taipei: Ministry of Health Department.

6. Liu SH (2009) A Portable Automatic Sphygmography and Pattern Recognition of Pulse Waveform. Yearbook of Chinese Medicine and Phamacy. Taipei: Ministry of Health Department.

\section{Your next submission with Juniper Publishers will reach you the below assets}

- Quality Editorial service

- Swift Peer Review

- Reprints availability

- E-prints Service

- Manuscript Podcast for convenient understanding

- Global attainment for your research

- Manuscript accessibility in different formats

(Pdf, E-pub, Full Text, Audio)

- Unceasing customer service

Track the below URL for one-step submission https://juniperpublishers.com/online-submission.php 